OPEN ACCESS

Edited by:

Tao Huang,

Shanghai Institute of Nutrition and Health (CAS), China

Reviewed by:

Ram Vinay Pandey,

Karolinska University Hospital,

Sweden

Luca Ermini,

Luxembourg Institute of Health, Luxembourg

Uday Shanker Evani,

New York Genome Center, United States

Ying Cai,

Albert Einstein College of Medicine,

United States

${ }^{*}$ Correspondence:

Yu Lu

samuelluyu@163.com

Hui Guo

guohui@sklmg.edu.cn

Fengxiao Bu

bufengxiao@wchscu.cn

Specialty section:

This article was submitted to

Computational Genomics,

a section of the journal

Frontiers in Genetics

Received: 08 January 2022

Accepted: 10 February 2022

Published: 03 March 2022

Citation:

Liu S, Zeng Y, Wang C, Zhang $Q$, Chen $M$, Wang $X$, Wang $L, L u Y$, Guo $H$ and Bu F (2022) seGMM: A New Tool

for Gender Determination From

Massively Parallel Sequencing Data.

Front. Genet. 13:850804.

doi: 10.3389/fgene.2022.850804

\section{seGMM: A New Tool for Gender Determination From Massively Parallel Sequencing Data}

\author{
Sihan Liu ${ }^{1}$, Yuanyuan Zeng ${ }^{2}$, Chao Wang ${ }^{1}$, Qian Zhang ${ }^{1}$, Meilin Chen ${ }^{1}$, Xiaolu Wang ${ }^{1}$, \\ Lanchen Wang ${ }^{1}, \mathrm{Yu} \mathrm{Lu}^{1 *}, \mathrm{Hui} \mathrm{Guo}^{3 *}$ and Fengxiao $\mathrm{Bu}^{1 *}$ \\ ${ }^{1}$ Institute of Rare Diseases, West China Hospital of Sichuan University, Chengdu, China, ${ }^{2}$ School of Medicine, National Institute for \\ Data Science in Health and Medicine, Xiamen University, Xiamen, China, ${ }^{3}$ Center for Medical Genetics and Hunan Provincial Key \\ Laboratory of Medical Genetics, School of Life Sciences, Central South University, Changsha, China
}

In clinical genetic testing, checking the concordance between self-reported gender and genotype-inferred gender from genomic data is a significant quality control measure because mismatched gender due to sex chromosomal abnormalities or misregistration of clinical information can significantly affect molecular diagnosis and treatment decisions. Targeted gene sequencing (TGS) is widely recommended as a first-tier diagnostic step in clinical genetic testing. However, the existing gender-inference tools are optimized for whole genome and whole exome data and are not adequate and accurate for analyzing TGS data. In this study, we validated a new gender-inference tool, seGMM, which uses unsupervised clustering (Gaussian mixture model) to determine the gender of a sample. The seGMM tool can also identify sex chromosomal abnormalities in samples by aligning the sequencing reads from the genotype data. The seGMM tool consistently demonstrated $>99 \%$ gender-inference accuracy in a publicly available 1,000-gene panel dataset from the 1,000 Genomes project, an in-house 785 hearing loss gene panel dataset of 16,387 samples, and a 187 autism risk gene panel dataset from the Autism Clinical and Genetic Resources in China (ACGC) database. The performance and accuracy of seGMM was significantly higher for the targeted gene sequencing (TGS), whole exome sequencing (WES), and whole genome sequencing (WGS) datasets compared to the other existing gender-inference tools such as PLINK, seXY, and XYalign. The results of seGMM were confirmed by the short tandem repeat analysis of the sex chromosome marker gene, amelogenin. Furthermore, our data showed that seGMM accurately identified sex chromosomal abnormalities in the samples. In conclusion, the seGMM tool shows great potential in clinical genetics by determining the sex chromosomal karyotypes of samples from massively parallel sequencing data with high accuracy.

Keywords: massively parallel sequencing data, Gaussian mixture model, gender, sex chromosomal abnormality, aneuploidy 


\section{INTRODUCTION}

The next-generation sequencing (NGS) technology has revolutionized human biology and medicine in the last decade. NGS is routinely used in clinical genetic testing for molecular diagnosis of hereditary disorders, infectious diseases, and immune disorders, non-invasive prenatal genetic testing, and personalized precision medicine, especially for cancer patients (Phillips and Douglas, 2018; Phillips et al., 2020). Clinical genetic testing is a diagnostic tool that involves genome sequencing to identify pathogenic gene mutations (genetic variants) in human diseases (McPherson, 2006). This may involve targeted gene sequencing (TGS) of single or multiple genes, whole exome sequencing (WES), or whole genome sequencing (WGS) (Di Resta et al., 2018). TGS is highly accurate, robust, and costeffective. Therefore, TGS has been used for the diagnosis of several human diseases including hearing loss, vision loss, cardiovascular disorders, neurologic disorders, cancer risk, and renal disorders (Lin et al., 2012; Saudi Mendeliome, 2015).

Parallelized TGS analysis of large patient cohorts requires rigorous quality control (QC) and preprocessing to identify the pathogenic gene variants (Lee et al., 2017). Verification of the concordance between self-reported gender and genetically inferred gender is an essential QC step because misregistration of clinical information, sample swaps, sample pollution, or sex chromosomal abnormalities can result in wrong conclusions and affect treatment decisions (Taylor et al., 2015; Webster et al., 2019). Sex chromosomal abnormalities are reported in approximately 1 in 448 newborn children (Nielsen and Wohlert, 1990). Therefore, there is a higher probability of gender inconsistencies in larger cohorts. Cytogenetic karyotyping is the gold standard method for confirming the gender of an individual and identifying chromosomal abnormalities. The highly conserved sex chromosomal marker gene, amelogenin, is widely used for identifying gender using short tandem repeat (STR) typing (Thangaraj et al., 2002; Ma et al., 2012). The 6 bp deletion within intron 1 of the amelogenin gene in the $\mathrm{X}$ chromosome is used to distinguish the PCR amplified products of the amelogenin gene in the $\mathrm{X}$ and $\mathrm{Y}$ chromosomes (Sullivan et al., 1993). However, these methods are time- and laborconsuming.

Several computational tools such as PLINK, seXY, and XYalign, have been developed for gender inference based on genome-wide WES or WGS data. PLINK inferred gender by calculating $\mathrm{F}$ coefficients from the genotyping array data using $\mathrm{X}$ chromosome homozygosity/heterozygosity rates; samples with $\mathrm{F}$ coefficient values of more than 0.8 were designated as males and samples with F coefficient values of less than 0.2 were considered as females (Purcell et al., 2007). The seXY tool is based on the logistic regression model and identifies gender by considering $\mathrm{X}$ chromosome heterozygosity and Y chromosome missingness in the genotyping array data (Qian et al., 2017). XYalign tool identifies gender from both WES and WGS datasets by extracting the read counts mapped to the sex chromosomes and calculating the ratio of $\mathrm{X}$ and $\mathrm{Y}$ read counts in a scatter plot (Webster et al., 2019). However, none of these tools are optimized for analyzing TGS panel data, which contains significantly reduced information compared to the whole genomic or exome data. As shown in Table 1, the performance of the existing tools was not satisfactory in reporting gender using the TGS data. Furthermore, sex chromosomal abnormalities were not clearly identified by the PLINK, seXY, and XYalign tools. Few studies reported the sex chromosomal abnormalities of individuals based on the ratio of sequencing reads that were mapped to the $\mathrm{X}$ and $\mathrm{Y}$ chromosomes from the genotyping array and WGS data (Bycroft et al., 2018; Turro et al., 2020). However, this methodology has not been automated. Therefore, there is an urgent need to construct highly accurate bioinformatics tools for gender inference from TGS data and reporting sex chromosomal abnormalities.

In this study, we verified the performance and accuracy of the new gender inference tool, seGMM, using both in-house and publicly available TGS, WES, and WGS datasets. The seGMM tool used unsupervised learning to integrate the information of the $\mathrm{X}$ and $\mathrm{Y}$ chromosomes from the TGS, WES, or WGS datasets and classified the samples into one of the six sex chromosomal karyotypes (XX, XY, XYY, XXY, XXX, and X).

\section{MATERIALS AND METHODS}

\section{Data}

We compared the performances of three existing genderinferring methods and seGMM using the TGS data from the following 3 datasets: 1) Dataset 1: exon-targeted sequencing data of 1,000 genes ( $34 \mathrm{X}$ chromosomal genes and two Y chromosomal genes) for a cohort of 110 males and 98 females from the 1,000 Genomes Project (Supplementary Table S1) (Genomes Project et al., 2010); 2) Dataset 2 (in-house): massive parallel sequencing of 785 deafness-related genes (eight genes in the $\mathrm{X}$ chromosome) for an in-house cohort of 8,805 males and 7,582 females; and 3) Dataset 3: targeted sequencing data of 187 autism risk genes (13 genes in the X chromosome) for a cohort of 42 females and 205 males from the Autism Clinical and Genetic Resources in China (ACGC) (Guo et al., 2018).

We also used the following two publicly available datasets (Supplementary Tables S2, S3) and one in-house dataset for analyzing the performance of seGMM in determining gender using WES and WGS data: 1) Dataset 4: exome sequencing data of 164 males and 118 females from the 1,000 Genomes Project (Genomes Project et al., 2015); 2) Dataset 5 (in-house): exome sequencing data of 1,257 males and 1,136 females; and 3) Dataset 6: high-coverage whole genome sequencing data of 11 males and 16 females from the 1,000 Genomes Project (Genomes Project et al,, 2015).

The publicly available BAM files were previously mapped to the reference genome (GRCh37) and directly used for downstream analyses. For the in-house datasets, Fastp was used to remove the adapters and low-quality reads, and the quality of sequencing data was evaluated using measures such as Q20, sequence duplication levels, coverage, and GC content (Chen et al., 2018). Clean DNA sequencing reads were mapped to the human reference genome (GRCh37) using the BWA-MEM algorithm (Li and Durbin, 2009). Duplicated reads in the BAM files from the public and in-house datasets were removed using 
TABLE 1 | Gender prediction accuracy of different methods for samples in dataset 1.

\begin{tabular}{|c|c|c|c|}
\hline Tools & $\begin{array}{l}\text { Accuracy for all samples } \\
(\%)\end{array}$ & $\begin{array}{l}\text { Accuracy for male samples } \\
\qquad(\%)\end{array}$ & $\begin{array}{c}\text { Accuracy for female } \\
\text { samples (\%) }\end{array}$ \\
\hline PLINK & 81.44 & 48.28 & 100 \\
\hline $\operatorname{seXY}$ & 62.5 & 45.45 & 81.63 \\
\hline XYalign & 98.08 & 100 & 95.92 \\
\hline seGMM & 99.52 & 100 & 98.98 \\
\hline
\end{tabular}

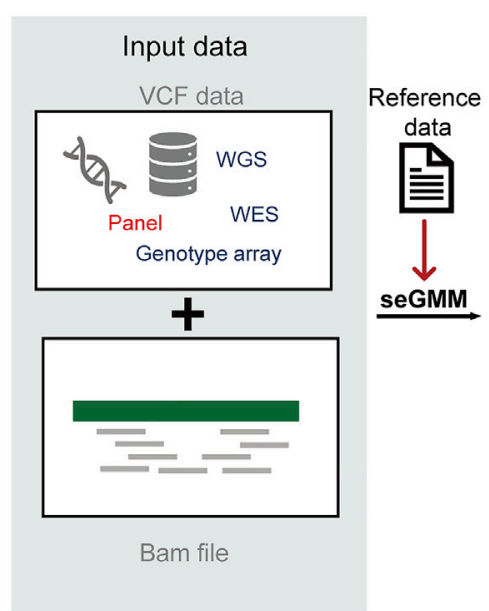

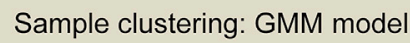
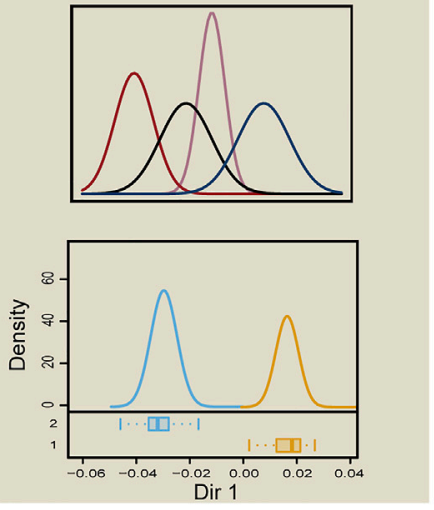

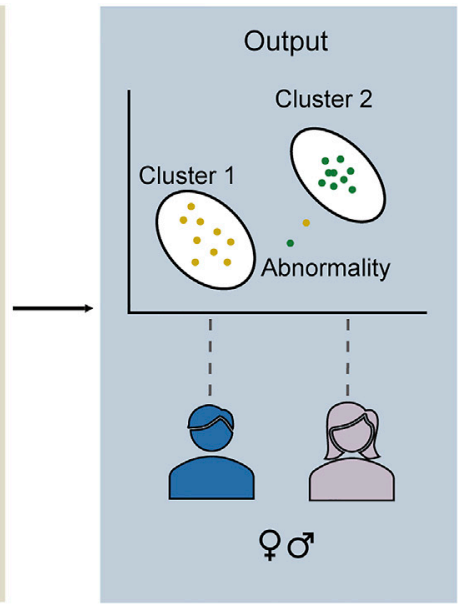

FIGURE 1 | Schematic diagram of seGMM. The seGMM tool automatically collects features from the input VCF and BAM files and builds the GMM model. The output of seGMM includes gender prediction results and identification of samples with abnormal sex chromosomes.

the sambamba tool (Tarasov et al., 2015). The variants were identified based on the Genome Analysis Toolkit best practices recommendations (McKenna et al., 2010) and filtered with VCFtools (Danecek et al., 2011) using parameters such as missing data in more than $50 \%$ of samples, minor allele count $<3$, overall SNP quality (QUAL) score $<30$, and read depth $<5$.

\section{Gender Inference Using seGMM}

The model for seGMM included five gender-associated features, namely, $\mathrm{X}$ chromosome heterozygosity $(\mathrm{XH})$, reads mapped to the $\mathrm{X}$ chromosome (Xmap), reads mapped to the $\mathrm{Y}$ chromosome (Ymap), the ratio of X/Y counts (XYratio), and the mean depth of the sexdetermining region of the Y chromosome (SRY) gene (SRY_dep). The seGMM tool computed $\mathrm{XH}$ as the fraction of all genotypes on the $\mathrm{X}$ chromosome with two different allele calls, excluding the missing genotypes. Xmap/Ymap was computed as the fraction of high-quality reads (mapq > 30) that mapped to the $\mathrm{X} / \mathrm{Y}$ chromosome divided by the total number of high-quality reads that mapped to the genome using the samtools algorithm (Li et al., 2009). XYratio was computed as the ratio of Xmap to Ymap (Xmap/ Ymap). SRY_dep was determined using the mosdepth tool (Pedersen and Quinlan, 2018). The seGMM tool allows the users to customize feature selection for the GMM model because different TGS panel designs may only provide some features. For example, if the gene panel contains only genes located on the $\mathrm{X}$ chromosome, the relevant features on the $\mathrm{X}$ chromosome (XH and Xmap) are extracted and put into the model for gender determination.
The features extracted from the BAM and VCF files were normalized to the same level using the scale function in R 4.1.2 ( $R$ Core Team., 2021). Then, the mclust (v.5.4.9) R package was used to perform model-based clustering with the expectationmaximization (EM) algorithm and the samples were classified into two clusters (Scrucca et al., 2016). The gender was inferred based on the cluster results for a group of samples. The outliers were identified when uncertainty (probability of being assigned to two different clusters) was greater than 0.1 . When a single sample was submitted, gender was inferred using the reference data that was analyzed with the same features as those in the seGMM model (Figure 1).

\section{Identifying Potential Sex Chromosomal Abnormalities in the Sequenced Samples}

We defined the gates to classify individual karyotypes. The distribution of Xmap and Ymap in the females and males of the large cohort was normal. The ratio of samples with sex chromosomal abnormalities was $0.022 \%$ (Nielsen and Wohlert, 1990). This data was in agreement with the empirical rule, which states that $99.7 \%$ of normally distributed data lies within 3 standard deviations (sd) of the mean. Hence, we defined the normal gates as mean \pm 3 sd. The fold changes in Xmap or Ymap values indicated sex chromosomal aneuploidy.

To identify sex chromosomal abnormalities in the samples, we first calculated the mean value and standard deviation values of 
Xmap (mean_xmap and sd_xmap) and Ymap (mean_ymap, and sd_ymap) in the genetically determined male and female samples. The values for the males and females were denoted as $m$ and $f$, respectively. The following six gates were then used to classify the karyotypes of individuals:

- XY Gate

O mean_xmap_m - 3 sd_xmap_m $<\mathrm{x}<$ mean_xmap_m +3 sd_xmap_m

O mean_ymap_m - 3 sd_ymap_m $<$ y $<$ mean_ymap_m +3 sd_ymap_m

- XYY gate:

O mean_xmap_m - 3 sd_xmap_m $<\mathrm{x}<$ mean_xmap_m +3 sd_xmap_m

$\mathrm{O}>2$ mean_ymap_m

- $\mathrm{XX}$ gate:

$O$ mean_xmap_f -3 sd_xmap_f $<\mathrm{x}<$ mean_xmap_f +3 sd_xmap_f

$O$ mean_ymap_f -3 sd_ymap_f $<$ y $<$ mean_ymap_f +3 sd_ymap_f

- XXY gate:

$O x>2$ mean_xmap_f

O mean_ymap_m - 3 sd_ymap_m $<$ y $<$ mean_ymap_m +3 sd_ymap_m

- $\mathrm{XXX}$ gate:

$O \mathrm{x}>3$ mean_xmap_f

O mean ymap_f -3 sd_ymap_f $<$ y $<$ mean_ymap_f +3 sd_ymap_f

- $\mathrm{X}$ gate:

$\mathrm{O} x<0.5$ mean_xmap_f

$O$ mean_ymap_f -3 sd_ymap_f $<y<$ mean_ymap_f +3 sd_ymap_f

\section{Comparing the Performance of seGMM With Other Existing Gender-Inference Methods}

The performance of seGMM was compared to PLINK 1.9, XYalign (v.1.1.6), and seXY (v.20170316). For PLINK 1.9, the pseudoautosomal region of the $\mathrm{X}$ chromosome was first split off with the parameter-split-x. Then, the parameter-check-sex was run without parameters. After reviewing the distribution of $\mathrm{F}$ estimates, the parameter-check-sex was rerun with parameters corresponding to the empirical gap. XYalign was performed following the method described in the original literature. The CHROM_STATS module was used to obtain the depths of chromosomes 1, X, and $\mathrm{Y}$. The depths of $\mathrm{X}$ and $\mathrm{Y}$ chromosomes were normalized relative to the depth of chromosome 1. Then, a scatter plot of normalized X and Y chromosomes depth was plotted to assess gender in samples. Gender of the samples was inferred with seXY using the X.ped and Y.ped data that was derived from PLINK. The training dataset was provided by seXY. We expected to compare seGMM and other existing tools for all the six datasets. However, target gene panel data for datasets 2 and 3 did not contain genes on the $\mathrm{Y}$ chromosome. Therefore, the performance of XYalign and seXY was not available for these two datasets.

\section{STR Analysis for Verifying Gender}

The STR analysis was performed using the customized multiplex PowerPlex ${ }^{\circledR} 16$ System, which allowed co-amplification and fourcolor detection of amelogenin and other gene loci. The following primers were used for amplifying amelogenin: forward, 5' - GTTAGACGTGTGCTTCAACTTCAGCTATG AGGTAATTTTTC-3'; reverse, $5^{\prime}$ - ATCCGACGGTAG TGTCCAACCATCAGAGCTTAAACTGG- $3^{\prime}$. All genetic loci were amplified simultaneously in a single tube and analyzed in a single lane. One of the primers for the amelogenin gene was labeled with carboxyrhodamine (ROX). The amplicons were separated in the ABI 3730XL Genetic Analyzer and the data was extracted using GeneMapper ID v3.2. The gender was inferred according to the peaks for the amelogenin gene. If only one peak was observed for the amelogenin locus, the gender was designated as female. If two distinct peaks differing by $6 \mathrm{bp}$ were observed in the amelogenin locus, the gender was designated as male.

\section{Quantitative Determination of $Y$ Chromosome Copy Number}

Genomic DNA (gDNA) was extracted using the MagMAX High Purity Free DNA Separation Kit (Magen, China). DNA concentration of the samples was measured using the NanoDrop One spectrophotometer (Thermo Fisher Scientific, United States). The working concentration of all DNA samples was $20 \mathrm{ng} / \mu \mathrm{l}$. The primers targeting $S R Y$, zinc finger protein Y-linked $(Z F Y)$, and deleted in azoospermia 1 (DAZ1) genes were designed using the Primer-BLAST online tool to determine the Y chromosome copy number (Ye et al., 2012). RPP30 was used as the internal control. All the primers used in this study are listed in Supplementary Table S4. The qPCR reaction mix included $0.6 \mu \mathrm{l}$ of gDNA, $0.4 \mu \mathrm{l}$ of each primer, $5 \mu \mathrm{l}$ of $\mathrm{iTaq}^{\mathrm{TM}}$ Universal SYBR ${ }^{\circledR}$ Green Supermix (Bio-Rad, United States), and $3.6 \mu \mathrm{l}$ of double-distilled water. Each sample was analyzed with three replicates. The quantitative real-time PCR assay (RT-qPCR) was performed in the QuantStudio 5 Real-Time PCR system (Thermo Fisher Scientific) using the following conditions: initial hot start cycle at $98^{\circ} \mathrm{C}$ for 2 min followed by 40 cycles consisting of denaturation at $98^{\circ} \mathrm{C}$ for $10 \mathrm{~s}$, annealing at $60^{\circ} \mathrm{C}$ for $10 \mathrm{~s}$, and the final extension step of $30 \mathrm{~s}$ at $72^{\circ} \mathrm{C}$.

\section{RESULTS}

\section{The seGMM Tool Shows Better Performance Compared to Other Tools for the TGS Data}

The distribution of XH, Xmap, Ymap, and XYratio values for dataset $1(n=208)$ shown in Figures 2A-D. The accuracy of seGMM was $99.52 \%$ and none of the samples were outliers (Figure 2E; Table 1). The accuracy of seGMM in females and males was 98.98 and $100 \%$, respectively. The XYratio of one female sample (NA19054) resembled that of males and was incorrectly 


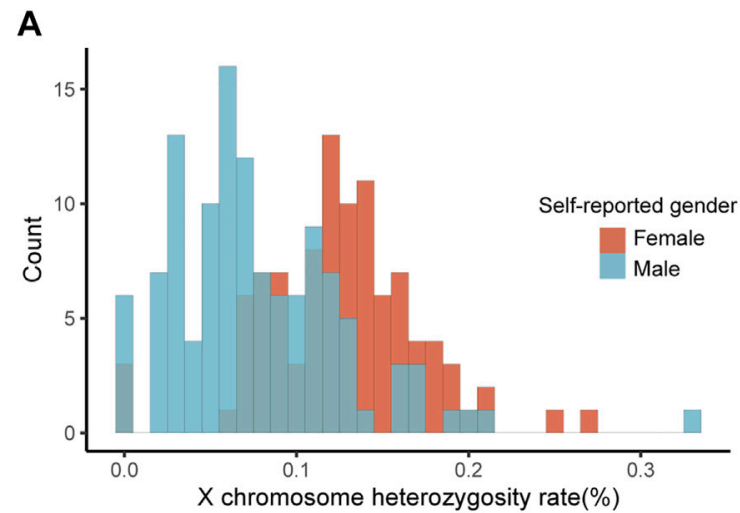

C

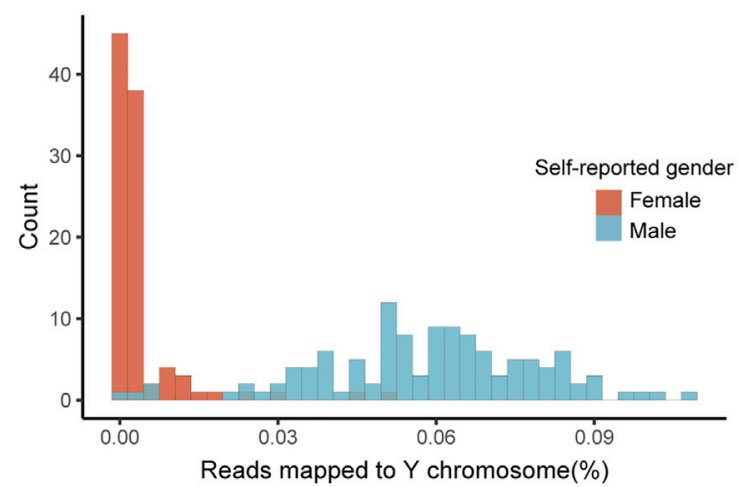

B

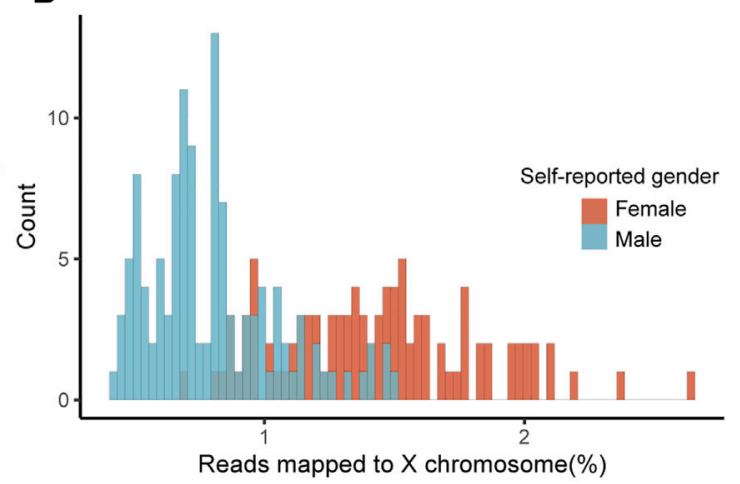

D

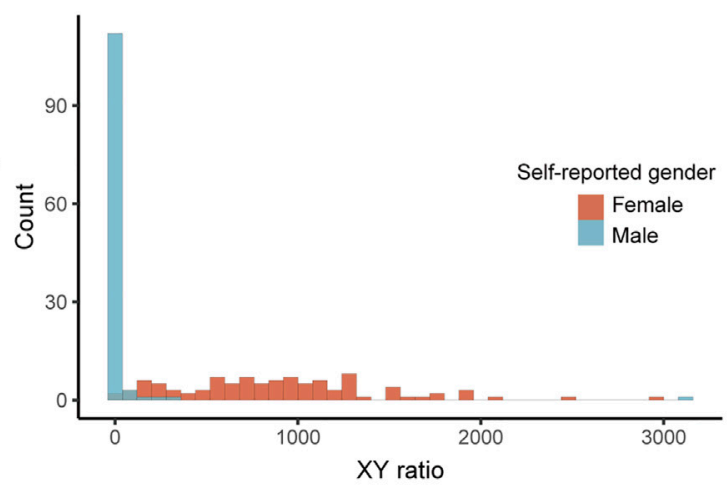

E

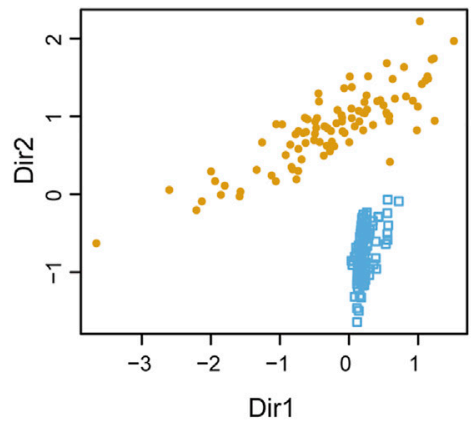

F

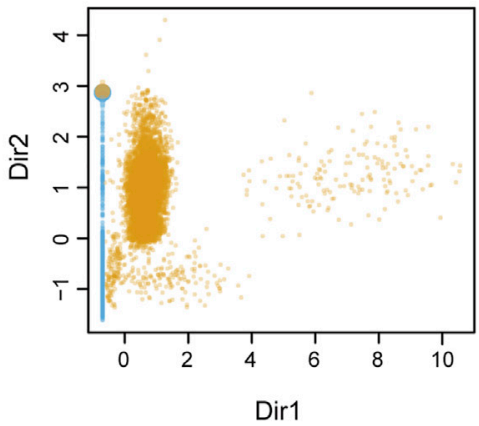

G

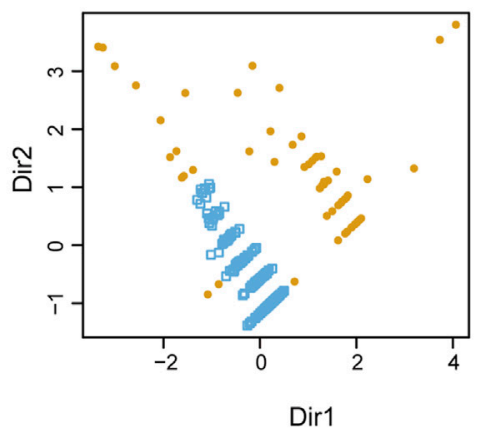

FIGURE 2 | The performance of seGMM in the TGS datasets. (A-D) Distribution of features collected from dataset 1. (E-G) Sample classification results of datasets 1, 2, and 3 based on seGMM. The colors represent different sample clusters. Dir1 and Dir2 represent the eigenvectors that specify the discriminant subspace generated from the features included in the GMM model.

classified as male by seGMM. The gender-inference performance of seGMM for dataset 1 was superior to PLINK, seXY and XYalign. The PLINK tool analysis showed that the F coefficients for the dataset 1 samples ranged from 0 to 0.9 and gap of $F$ coefficients was not observed (Supplementary Figure S1). The accuracy of PLINK was $81.44 \%$ by running -check-sex without parameters. The accuracy of seXY for the dataset 1 was only $62.5 \%$ (Table 1). XYalign does not directly indicate predicted gender. Therefore, plotting the normalized sequence depth of the sex chromosomes and cluster samples along two ellipses using the stat_ellipse function resulted in a confidence level of $99.99 \%$. XYalign plot showed that one female sample was located along with the male samples and three female samples were located between the two ellipses. Hence, the predicted gender of these four female samples was ambiguous (Supplementary Figure S2) and the accuracy of XYalign is $98.08 \%$. 
TABLE 2 | Gender prediction accuracy of different methods for samples in datasets 2 and 3 .

\begin{tabular}{lcc}
\hline Methods & Dataset 2 & Dataset 3 \\
\hline PLINK & 87.10 & 38.87 \\
SeGMM & 99.92 & 92.31
\end{tabular}

The performance of seGMM for the target gene panel data was validated using dataset $2(n=16,387)$ and dataset $3(n=$ 247). The read counts, base quality, and GC distribution of the sequencing data of all the 16,387 subjects in dataset 2 was assessed. The average total number of sequence read per sample was 10.72 million. The average quality score for all bases was above 30 and the average GC content was $50.11 \%$ per subject. The average targeted sequence coverage was $90.43 \%$, and unique mapping rate of each sample was $99.26 \%$. We identified 16,988 variants in eight genes located on the $\mathrm{X}$ chromosome. Because the target gene panel for dataset 2 did not contain genes on the $\mathrm{Y}$ chromosome, we only used $\mathrm{XH}$ and Xmap to analyze the performance of the seGMM model. XH and Xmap plots showed distinct clusters for males and females (Supplementary Figure S3). The overall accuracy of seGMM and PLINK was 99.92 and $87.10 \%$, respectively (Figure 2F; Table 2). The accuracy of seGMM in females and males was both $99.98 \%$. One self-reported female sample (HL001200) and two self-reported male samples (CTRL-002692 and CTRL-002753) were misclassified. Therefore, we performed STR analysis with the sex chromosome marker gene, amelogenin, to verify the gender of these three ambiguous samples. All these three samples were identified as males because two distinct peaks were observed with a difference of $6 \mathrm{bp}$ for the amelogenin gene (Figures 3A-C). CTRL-002692 and CTRL-002753 were misclassified because all other male samples had a $\mathrm{XH}$ value of 0 , while these two samples had a non-zero value $\left(\mathrm{XH}_{\mathrm{CTRL}-002692}=0.0025\right.$; $\mathrm{XH}_{\mathrm{CTRL}-002753}=0.0046$ ), which could be caused by the individual variation in targeting region.

The overall accuracy of seGMM for dataset 3 was $92.31 \%$ (97.56\% for females and $84.88 \%$ for males, Figure $2 \mathrm{G}$ and Table 2). The accuracy of PLINK was only $38.87 \%$ for dataset 3. The performances of seGMM and PLINK were significantly better for datasets 1 and 2 compared to dataset 3 because the number of X chromosome SNPs (81) were lower and sequencing data for the $\mathrm{Y}$ chromosome was absent in dataset 3, thereby affecting the distribution of $\mathrm{XH}$ values from the male and female samples (Supplementary Figure S4A). In contrast to PLINK, seGMM collected additional information for the reads mapped to the $\mathrm{X}$ chromosome, thereby enabling better separation between the female and male samples (Supplementary Figure S4B). Furthermore, we assessed the performance of seGMM using features only extracted from the $\mathrm{X}$ chromosome in dataset 1 . The seGMM tool showed that 59 samples were outliers and the accuracy for the remaining samples was only $84.56 \%$. We then evaluated the computation time of different methods using 1 core, 10 cores and 20 cores on a server with $64 \operatorname{Intel}(\mathrm{R}) \mathrm{Xeon}(\mathrm{R})$ CPU E7-8895 v3 at $2.60 \mathrm{GHz}$. The analysis time for the seGMM tool was longer than PLINK and seXY because it collected additional features such as reads mapped to the $\mathrm{X}$ and $\mathrm{Y}$ chromosomes. Moreover, the analysis time for seGMM with 1 core was longer than XYalign and 10 times faster than XYalign with 20 cores (Supplementary Table S5).

\section{The seGMM Tool Shows Better Accuracy Than Other Known Tools for the WES and WGS Data}

We then evaluated the performance of the seGMM tool for the WES and WGS data. First, we analyzed the publicly available WES data (dataset 4). The accuracy of seGMM was $100 \%$ for the samples in dataset $4(n=282)$ (Table 3 and Supplementary Figure S5). The accuracy of PLINK and seXY was also $100 \%$. The accuracy of XYalign was 99.65\% (Supplementary Figure S6).

Next, we analyzed the in-house WES data (dataset $5, n=2,393$ ) using seGMM and other tools. In dataset 5 , the average number of sequencing reads per sample was 114.43 million. The average Q20, Q30 and GC content of the reads per subject was $97.36 \%, 93.21 \%$, and $51.23 \%$, respectively. Furthermore, the average unique mapping rate for each individual sample was $99.92 \%$. We identified 89,273 variants on the X chromosome and 4,866 variants on the $\mathrm{Y}$ chromosome. The concordance between inferred gender and self-reported gender using the seGMM tool based on the five features for the in-house WES dataset 5 was 99.75\% (99.76\% for males and $99.74 \%$ for females, Figure 4A, Supplementary Figure S7). Six mismatched samples (HL-005584, HL-006009, HL-006904, HL-007335, HL-007935 and HL-012246) were identified by comparing SNP-inferred gender and selfreported gender. This indicated misregistration of clinical information for some samples. Therefore, we performed STR analysis to validate the gender of these six samples. Three samples were classified as females because they showed only one peak for the amelogenin locus, whereas the remaining three samples showed two distinct peaks with a difference of $6 \mathrm{bp}$ and were classified as males (Table 4). The results demonstrated that the actual accuracy of seGMM prediction was $100 \%$. We also evaluated the correlation between age and reads mapped to the $\mathrm{Y}$ chromosome in the male samples (Supplementary Figure S8A) and reads mapped to the $\mathrm{X}$ chromosome in the female samples from the in-house WES dataset 5 (Supplementary Figure S8B). The results showed significant negative correlation ( $p=6.899 \mathrm{e}-09$; correlation coefficient: -0.17 ) between reads mapped to the $Y$ chromosome and age, thereby indicating loss of $\mathrm{Y}$ chromosome during aging.

We then compared the performances of PLINK, seXY and $\mathrm{XYalign}$ for dataset 5 using the corrected gender information. The accuracy of PLINK was $99.79 \%$ with five mismatched samples (HL-033182, HL-020292, HL-011500, HL-019211 and HL012554) (Table 3). The accuracy of XYalign was $99.91 \%$ with two mismatched samples (HL-009389 and HL-012554). Overall, six samples were mismatched, as predicted by PLINK and XYalign. STR analysis showed that the gender of these samples was consistent with their self-reported gender and matched the predicted results of seGMM analysis (Table 4). Furthermore, the accuracy of seXY was $49.23 \%$. The loss of 


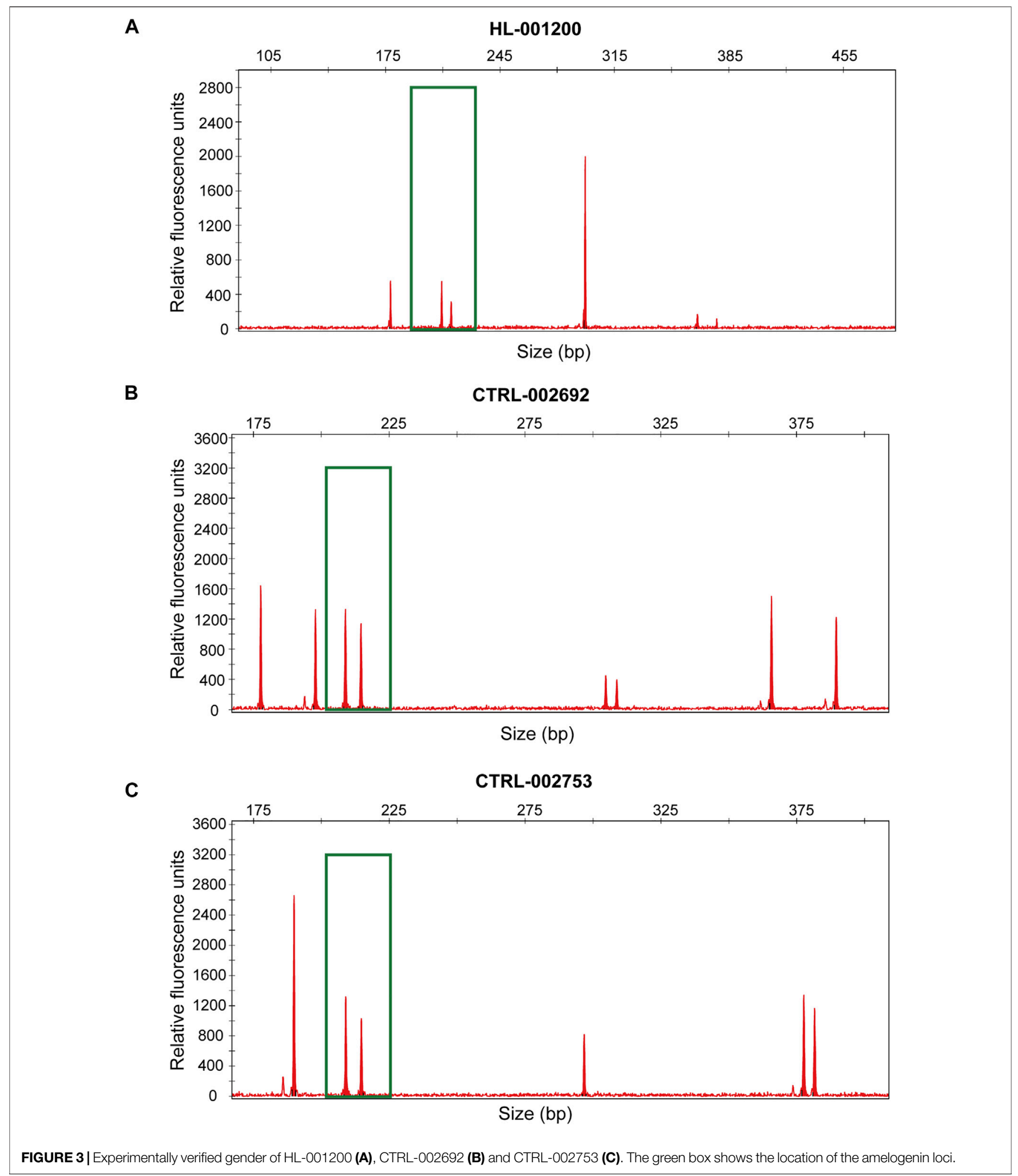

accuracy in seXY for dataset 5 was because the distribution of $\mathrm{Y}$ chromosome missingness in the male and female samples were confounded (Supplementary Figure S9). Finally, the performances of these tools were assessed using the WGS data (dataset $6, n=27$ ). The accuracy of all tools was $100 \%$ for dataset 6 (Table 3). 
TABLE 3| Gender prediction accuracy of different methods for the WES and WGS datasets.

\begin{tabular}{lcccc} 
Datasets & PLINK & XYalign & seXY & seGMM \\
\hline 1000G phase3 WES data & 100 & 99.65 & 100 & 100 \\
1000G phase3 high quality WGS data & 100 & 100 & 100 & 100 \\
In-house WES data & 99.79 & 99.91 & 49.23 & 100 \\
\hline
\end{tabular}

\section{The seGMM Tool Identifies Samples With Sex Chromosomal Abnormalities}

The seGMM tool can identify six sex chromosomal karyotypes (XX, XY, XYY, XXY, XXX, and X) using Xmap and Ymap. In a large cohort, the distribution of Xmap and Ymap was normal in females and males. The Xmap or Ymap values of samples with sex chromosome abnormalities such as XYY and XXY were significantly different and were recognized as outliers compared to samples with normal sex chromosomes. Three samples in dataset 5 (HL-029620, HL-009382 and HL-019110) were classified as the XYY karyotype. In dataset 5, the average rate of reads mapping to the $\mathrm{X}$ and $\mathrm{Y}$ chromosomes in the female samples were $0.035 \pm 0.0020$ and $1.61 \mathrm{e}-05 \pm 3.36 \mathrm{e}-05$, respectively, and $0.018 \pm 0.0011$ and $0.00067 \pm 0.00014$, respectively, for the male samples. The rate of reads mapping to the $\mathrm{Y}$ chromosome for the three outlier samples was twice as high as the mean value of Ymap in all the male samples $\left(\right.$ Ymap $_{\mathrm{HL}-029620}=0.0015$, Ymap ${ }_{\mathrm{HL}-009382}=$ 0.0018 , and Ymap $\left.{ }_{\mathrm{HL}-019110}=0.0015\right)$, thereby suggesting a XYY karyotype by seGMM (Figure 4B). Furthermore, although HL009389 and HL-012554 samples were located close together in the middle of the plot, they were correctly predicted by seGMM as female and male, respectively (Figure 4B). This is because features such as Xmap and SRY_dep, which are not shown in Figure 4B, clearly separated all female and male samples (Supplementary Figure S10). This demonstrated the significance of incorporating key features to improve the accuracy of the gender prediction model. In the other datasets, sex chromosomal abnormalities were not identified.

Next, we evaluated the accuracy of the data-based sex chromosome karyotype of these three samples by analyzing the copy number ratios of $\mathrm{Y}$ chromosome-specific genes ( $S R Y$, ZFY and DAZ1) by RT-qPCR. We used HL-007935 and HL012246 samples as controls for females and males based on the STR analysis results. The copy number ratio for normal females was 0 . The copy number ratio for normal male samples was 1 . We analyzed the copy number ratios of HL-029620, HL-009382 and HL-019110 samples in dataset 5 and found that the copy number ratio of HL-019110 was 2 (Figure 5). This confirmed that the karyotype for the HL-019110 sample was XYY.

\section{DISCUSSION}

In this study, we characterized the performance of the new gender inference tool, seGMM, in comparison with the other established gender inference tools using NGS data, especially TGS panel data. The seGMM tool used unsupervised clustering to classify samples based on $\mathrm{X}$ and $\mathrm{Y}$ sex chromosomal features. The performance and accuracy of the seGMM tool were significantly better than other existing gender inference tools using TGS, WES, and WGS data. Furthermore, seGMM accurately predicted six different sex chromosomal karyotypes, including those with sex chromosome abnormalities. The mean and standard deviation values of Xmap and Ymap were used to determine potential sex chromosome aneuploidy in the male and female samples by seGMM. Previous studies have identified sex chromosomal aneuploidy in samples by measuring the intensities of $\mathrm{X}$ and $\mathrm{Y}$ chromosomes (Bycroft et al., 2018; Turro et al., 2020). A similar strategy was incorporated into the seGMM tool and used to validate a sample with sex
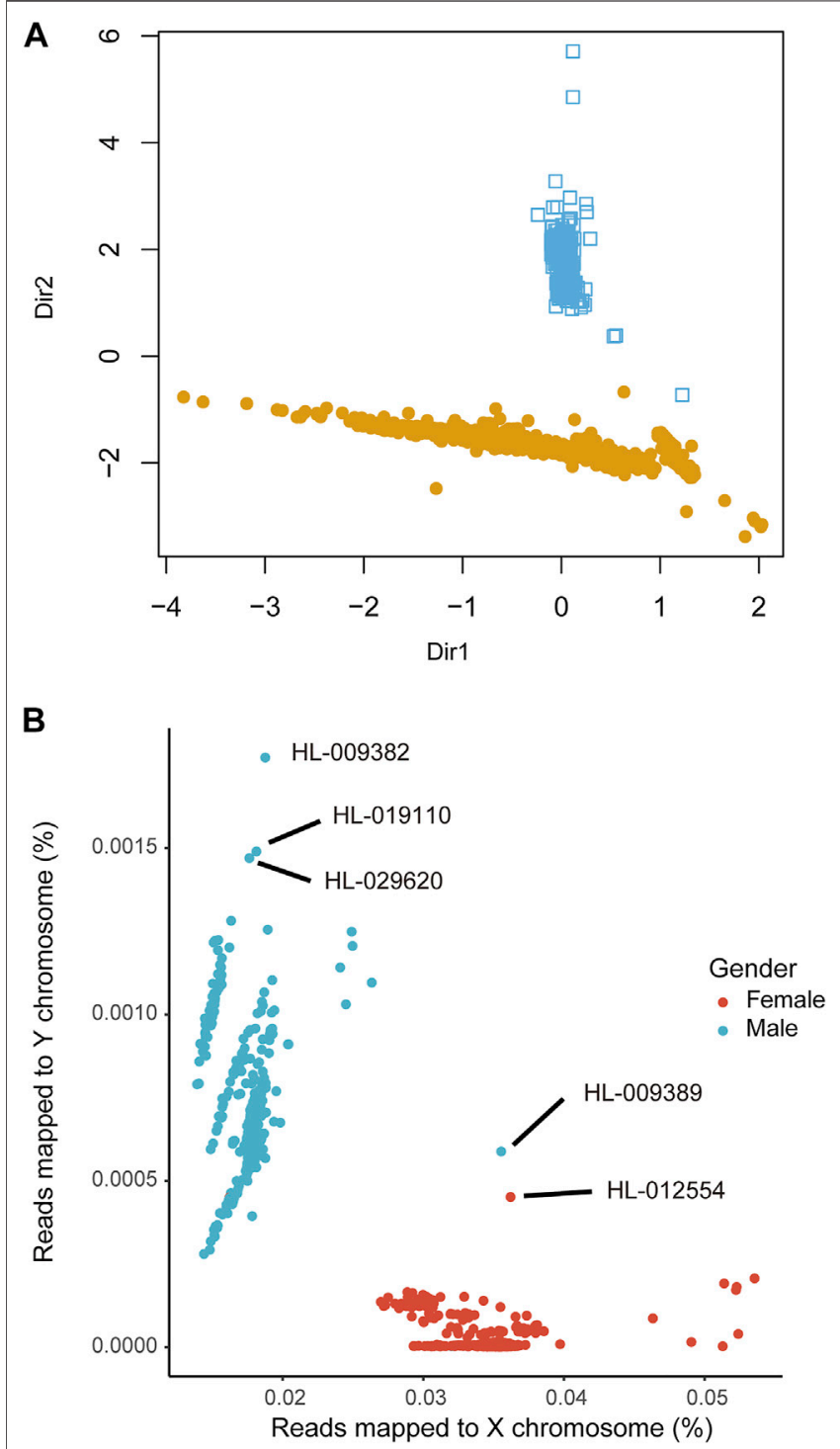

FIGURE 4 | The prediction accuracy of seGMM in inferring the gender of samples from the in-house WES dataset. (A) Sample clustering results of seGMM. The colors represent different sample clusters. Dir1 and Dir2 represent eigenvectors that specify the discriminant subspace generated from the features included in the GMM model. (B) Scatter plot shows the reads mapped to the $X$ and $Y$ chromosomes. As shown, we identified three samples (HL-029620, HL-009382 and HL-019110) with XYY sex chromosome karyotypes. 
TABLE 4 | Experimental verification of gender prediction results for samples in the in-house WES data.

\begin{tabular}{|c|c|c|c|c|}
\hline Sample ID & $\begin{array}{c}\text { Size of PCR } \\
\text { products in the } \\
\text { amelogenin loci (bp) }\end{array}$ & Self-reported gender & seGMM inferred gender & $\begin{array}{c}\text { Experimentally } \\
\text { validated gender }\end{array}$ \\
\hline HL-005584 & $\begin{array}{c}209.15 \\
-\end{array}$ & Male & Female & Female \\
\hline HL-006009 & $\begin{array}{c}209.06 \\
-\end{array}$ & Male & Female & Female \\
\hline HL-006904 & $\begin{array}{c}209.04 \\
214.8\end{array}$ & Female & Male & Male \\
\hline HL-007335 & $\begin{array}{l}209.06 \\
214.85\end{array}$ & Female & Male & Male \\
\hline HL-007935 & $\begin{array}{c}209.11 \\
-\end{array}$ & Male & Female & Female \\
\hline HL-012246 & $\begin{array}{l}209.18 \\
214.92\end{array}$ & Female & Male & Male \\
\hline HL-033182 & $\begin{array}{c}209.02 \\
-\end{array}$ & Female & Female & Female \\
\hline HL-020292 & $\begin{array}{c}209.19 \\
-\end{array}$ & Female & Female & Female \\
\hline HL-011500 & $\begin{array}{l}209.25 \\
215.07\end{array}$ & Male & Male & Male \\
\hline HL-019211 & $\begin{array}{l}209.19 \\
215.04\end{array}$ & Male & Male & Male \\
\hline HL-009389 & $\begin{array}{c}209.27 \\
-\end{array}$ & Female & Female & Female \\
\hline HL-012554 & $\begin{array}{l}209.26 \\
215.03\end{array}$ & Male & Male & Male \\
\hline
\end{tabular}

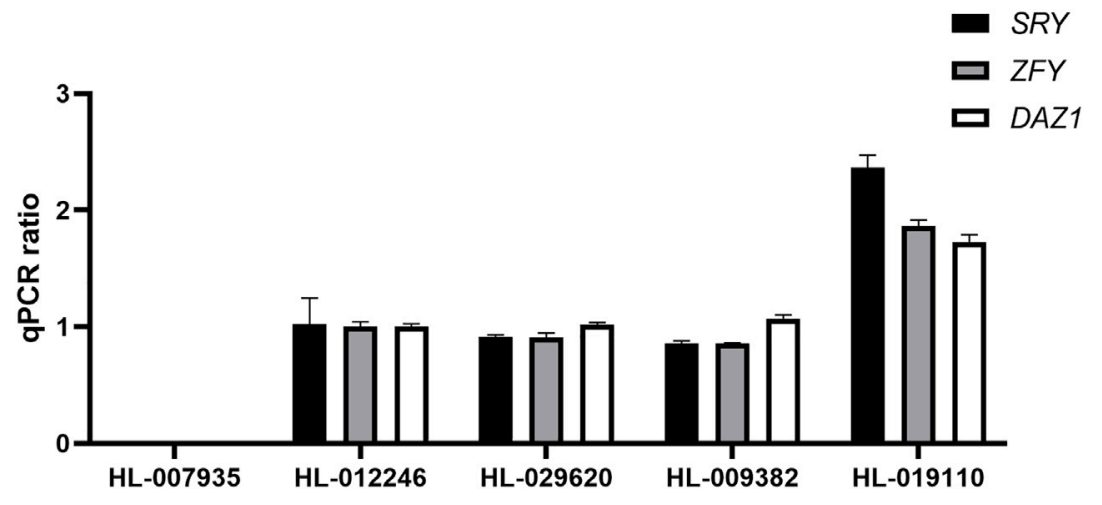

FIGURE 5 | Quantitative determination of $Y$ chromosome copy number.

chromosome karyotype XYY in the in-house WES dataset. Samples with sex chromosomal abnormalities may result in false calling of the genotype. This can affect identification of pathogenic variants in the sex chromosomes. Therefore, samples with sex chromosome abnormalities should be removed or recalled genotypes to ensure accuracy of the clinical diagnosis.

The seGMM tool applies unsupervised learning algorithm to infer gender of samples from the TGS panel data to overcome the pitfalls of existing tools. The TGS panel consists of a select set of genes with known or suspected association with the disease under study. The advantage of TGS in clinical genetic testing includes high sequencing depth of the genes of interest, which allows identification of rare and causative variants (Eggers et al., 2016;
Bewicke-Copley et al., 2019). The data size of TGS depends on the number of genes included in the panel and the methods used for targeted sequencing including target enrichment by hybridization capture and amplicon sequencing. Hence, the number of variants and sequencing depth of the $\mathrm{X}$ and $\mathrm{Y}$ chromosomes varies for different TGS panels. The accuracy of existing methods in inferring gender using TGS data is unsatisfactory because the algorithms are either based on a data-dependent threshold or supervised learning on a fixed sample set (Purcell et al., 2007; Qian et al., 2017). PLINK uses a data-dependent threshold strategy that determines gender by computing the $\mathrm{F}$ coefficients based on the observed and expected number of homozygous markers and requires reasonable minor allele frequency estimates. However, variants 
detected in the TGS datasets tend to have lower minor allele frequency and the number of variants detected in the $\mathrm{X}$ chromosome are limited. Therefore, $\mathrm{F}$ coefficient of the male and female samples based on the TGS data is ambiguous. Furthermore, the logistic regression classifier for the seXY tool was based on GWAS data collected from prostate cancer and ovarian cancer samples, and was not suitable for TGS panels because the distribution of $\mathrm{X}$ chromosome heterozygosity and $\mathrm{Y}$ chromosome missingness varied between the TGS panel dataset and the training dataset. In contrast, seGMM applied a Gaussian mixture model to infer gender. Therefore, the performance and accuracy of seGMM were higher for data with different covariance structures and were adaptable to include fresh samples.

Our study also demonstrated that the gender-inference accuracy of seGMM improved when the information from both $\mathrm{X}$ and $\mathrm{Y}$ chromosomes was available. For example, the accuracy of seGMM for dataset 1 was $84.56 \%$ when the data included only $\mathrm{X}$ chromosomal features, but the accuracy increased to $99.52 \%$ upon adding $\mathrm{Y}$ chromosomal features. Moreover, the accuracy of seGMM was lower for male samples compared to female samples in datasets 2 and 3 because the sequencing data did not contain information on genes in the $\mathrm{Y}$ chromosome. Our data also suggested that addition of probes that target unique regions of the Y chromosome such as the SRY exon, which is involved in typical male sex development (Gubbay et al., 1990; Parma and Radi, 2012), is helpful for inferring genders using the TGS panel data.

DNA sequencing data from the lymphoblastoid cell lines (LCLs) established from the EBV-infected peripheral blood mononuclear cells (PBMCs) may confound the prediction of sex chromosomal karyotypes. A previous study demonstrated that EBV transformation adversely affected the genomic DNA stability; mosaic loss of X chromosome was observed in 7\% (2/29) of the samples analyzed (Shirley et al., 2012). The false-positive rates due to EBV-induced mutations in LCLs may reduce the accuracy of predicting the sex chromosomal karyotypes. The majority of samples in the 1000G WES data were derived from LCLs, but we did not identify any sample in this dataset with abnormal sex chromosomal aneuploidy. The box plots of reads mapped to the $\mathrm{Y}$ chromosome showed a much lower value for one male sample (NA12413) compared to the others, thereby indicating potential loss of chromosome Y (Supplementary Figure S11). However, we could not confirm if the loss of Y chromosome was due to LCLs or as a result of authentic sex chromosome abnormalities since experimental validation is required for further analysis.

A few critical considerations are necessary while applying seGMM. First, seGMM is not applicable when the targeted sequencing data does not include genes located on the $\mathrm{X}$ and Y chromosomes. Secondly, seGMM requires a sufficient sample size to train an accurate model. Therefore, prediction accuracy should be enhanced for small sample datasets by including reference data (using -reference function parameter). We have provided two reference datasets that were generated from the 1000G WES and WGS datasets. In addition, samples sequenced with the same version of TGS panel can be used to build a user's own reference to maximize the accuracy of gender prediction. When applying seGMM, the experimental and analytical methods between reference data and testing data need to be consistent to prevent bias. Thirdly, parallel computing (using -num_threshold function parameter) is recommended to speed up the analysis since the seGMM tool collects more features than the other existing tools. Lastly, the use of Empirical Rule to classify individual karyotypes improves the recall rate, but may magnify false positives rate, as has been reported in previous study using this strategy (Turro et al., 2020). In addition, many factors may contribute to false positive predicition results, including the copy number variations such as large deletions or insertions on the sex chromosomes or genetic chimerism. Therefore, to overcome this limitation, karyotyping of predicted abnormal samples is recommended to confirm the sample karyotype.

In conclusion, we demonstrate that the performance and accuracy of seGMM, a new tool to infer sex chromosomal karyotypes based on a Gaussian mixture model, was significantly higher and satisfactory for TGS, WES, and WGS datasets, including those with samples containing sex chromosomal abnormalities compared to other existing tools. Hence, seGMM is a promising tool for inferring the gender of samples in TGS, WES, and WGS datasets.

\section{DATA AVAILABILITY STATEMENT}

The original contributions presented in the study are included in the article/Supplementary Material, further inquiries can be directed to the corresponding authors.

\section{ETHICS STATEMENT}

Written informed consent was obtained from the individual(s), and minor(s)' legal guardian/next of kin, for the publication of any potentially identifiable images or data included in this article.

\section{AUTHOR CONTRIBUTIONS}

SL developed the seGMM tool and drafted the manuscript; YZ, $\mathrm{QZ}$, and MC participated in data pre-processing and testing; $\mathrm{CW}$, $\mathrm{XW}$, and LW performed the experiments; YL and HG reviewed and revised the manuscript; $\mathrm{FB}$ designed and supervised the study; FB reviewed the manuscript; All authors contributed to the article and approved the final submitted version.

\section{FUNDING}

This work was financially supported by the $1 \cdot 3 \cdot 5$ project for disciplines of excellence, West China Hospital, Sichuan University.

\section{SUPPLEMENTARY MATERIAL}

The Supplementary Material for this article can be found online at: https://www.frontiersin.org/articles/10.3389/fgene.2022.850804/ full\#supplementary-material 


\section{REFERENCES}

Bewicke-Copley, F., Arjun Kumar, E., Palladino, G., Korfi, K., and Wang, J. (2019). Applications and Analysis of Targeted Genomic Sequencing in Cancer Studies. Comput. Struct. Biotechnol. J. 17, 1348-1359. doi:10.1016/j.csbj.2019.10.004

Bycroft, C., Freeman, C., Petkova, D., Band, G., Elliott, L. T., Sharp, K., et al. (2018). The UK Biobank Resource with Deep Phenotyping and Genomic Data. Nature 562 (7726), 203-209. doi:10.1038/s41586-018-0579-Z

Chen, S., Zhou, Y., Chen, Y., and Gu, J. (2018). Fastp: an Ultra-fast All-In-One FASTQ Preprocessor. Bioinformatics 34 (17), i884-i890. doi:10.1093/ bioinformatics/bty560

Danecek, P., Auton, A., Abecasis, G., Albers, C. A., Banks, E., DePristo, M. A., et al. (2011). The Variant Call Format and VCFtools. Bioinformatics 27 (15), 2156-2158. doi:10.1093/bioinformatics/btr330

Di Resta, C., Galbiati, S., Carrera, P., and Ferrari, M. (2018). Next-generation Sequencing Approach for the Diagnosis of Human Diseases: Open Challenges and New Opportunities. EJIFCC 29 (1), 4-14.

Eggers, S., Sadedin, S., van den Bergen, J. A., Robevska, G., Ohnesorg, T., Hewitt, J., et al. (2016). Disorders of Sex Development: Insights from Targeted Gene Sequencing of a Large International Patient Cohort. Genome Biol. 17 (1), 243. doi:10.1186/s13059-016-1105-y

Genomes Project, C., Abecasis, G. R., Altshuler, D., Auton, A., Brooks, L. D., Durbin, R. M., et al. (2010). A Map of Human Genome Variation from Population-Scale Sequencing. Nature 467 (7319), 1061-1073. doi:10.1038/ nature09534

Genomes Project, C., Auton, A., Brooks, L. D., Durbin, R. M., Garrison, E. P., Kang, H. M., et al. (2015). A Global Reference for Human Genetic Variation. Nature 526 (7571), 68-74. doi:10.1038/nature15393

Gubbay, J., Collignon, J., Koopman, P., Capel, B., Economou, A., Münsterberg, A., et al. (1990). A Gene Mapping to the Sex-Determining Region of the Mouse Y Chromosome Is a Member of a Novel Family of Embryonically Expressed Genes. Nature 346 (6281), 245-250. doi:10.1038/346245a0

Guo, H., Wang, T., Wu, H., Long, M., Coe, B. P., Li, H., et al. (2018). Inherited and Multiple De Novo Mutations in Autism/developmental Delay Risk Genes Suggest a Multifactorial Model. Mol. Autism 9, 64. doi:10.1186/s13229-018-0247-z

Lee, C., Bae, J. S., Ryu, G. H., Kim, N. K. D., Park, D., Chung, J., et al. (2017). A Method to Evaluate the Quality of Clinical Gene-Panel Sequencing Data for Single-Nucleotide Variant Detection. J. Mol. Diagn. 19 (5), 651-658. doi:10. 1016/j.jmoldx.2017.06.001

Li, H., and Durbin, R. (2009). Fast and Accurate Short Read Alignment with Burrows-Wheeler Transform. Bioinformatics 25 (14), 1754-1760. doi:10.1093/ bioinformatics/btp324

Li, H., Handsaker, B., Wysoker, A., Fennell, T., Ruan, J., Homer, N., et al. (2009). The Sequence Alignment/Map Format and SAMtools. Bioinformatics 25 (16), 2078-2079. doi:10.1093/bioinformatics/btp352

Lin, X., Tang, W., Ahmad, S., Lu, J., Colby, C. C., Zhu, J., et al. (2012). Applications of Targeted Gene Capture and Next-Generation Sequencing Technologies in Studies of Human Deafness and Other Genetic Disabilities. Hearing Res. 288 (12), 67-76. doi:10.1016/j.heares.2012.01.004

Ma, Y., Kuang, J.-Z., Zhang, J., Wang, G.-M., Wang, Y.-J., Jin, W.-M., et al. (2012). $Y$ Chromosome Interstitial Deletion Induced Y-STR Allele Dropout in AMELY-Negative Individuals. Int. J. Leg. Med 126 (5), 713-724. doi:10. 1007/s00414-012-0720-8

McKenna, A., Hanna, M., Banks, E., Sivachenko, A., Cibulskis, K., Kernytsky, A., et al. (2010). The Genome Analysis Toolkit: a MapReduce Framework for Analyzing Next-Generation DNA Sequencing Data. Genome Res. 20 (9), 1297-1303. doi:10.1101/gr.107524.110

McPherson, E. (2006). Genetic Diagnosis and Testing in Clinical Practice. Clin. Med. Res. 4 (2), 123-129. doi:10.3121/cmr.4.2.123

Nielsen, J., and Wohlert, M. (1990). Sex Chromosome Abnormalities Found Among 34,910 Newborn Children: Results from a 13-year Incidence Study in Arhus, Denmark. Birth Defects Orig Artic Ser. 26 (4), 209-223.

Parma, P., and Radi, O. (2012). Molecular Mechanisms of Sexual Development. Sex. Dev. 6 (1-3), 7-17. doi:10.1159/000332209

Pedersen, B. S., and Quinlan, A. R. (2018). Mosdepth: Quick Coverage Calculation for Genomes and Exomes. Bioinformatics 34 (5), 867-868. doi:10.1093/ bioinformatics/btx699
Phillips, K. A., and Douglas, M. P. (2018). The Global Market for Next-Generation Sequencing Tests Continues its Torrid Pace. J. Precis Med. 4.

Phillips, K. A., Douglas, M. P., and Marshall, D. A. (2020). Expanding Use of Clinical Genome Sequencing and the Need for More Data on Implementation. JAMA 324 (20), 2029-2030. doi:10.1001/jama.2020.19933

Purcell, S., Neale, B., Todd-Brown, K., Thomas, L., Ferreira, M. A. R., Bender, D., et al. (2007). PLINK: a Tool Set for Whole-Genome Association and Population-Based Linkage Analyses. Am. J. Hum. Genet. 81 (3), 559-575. doi:10.1086/519795

Qian, D. C., Busam, J. A., Xiao, X., O’Mara, T. A., Eeles, R. A., Schumacher, F. R., et al. (2017). seXY: a Tool for Sex Inference from Genotype Arrays. Bioinformatics 33 (4), btw696-563. doi:10.1093/bioinformatics/btw696

R Core Team (2021). R: A Language and Environment for Statistical Computing. Vienna, Austria: R Foundation for Statistical Computing. URL https://www.Rproject.org/.

Saudi Mendeliome, G. (2015). Comprehensive Gene Panels Provide Advantages over Clinical Exome Sequencing for Mendelian Diseases. Genome Biol. 16, 134. doi:10.1186/s13059-015-0693-2

Scrucca, L., Fop, M., Murphy, T. B., and Raftery, A. E. (2016). Mclust 5: Clustering, Classification and Density Estimation Using Gaussian Finite Mixture Models. R. J. 8 (1), 289-317. doi:10.32614/rj-2016-021

Shirley, M. D., Baugher, J. D., Stevens, E. L., Tang, Z., Gerry, N., Beiswanger, C. M., et al. (2012). Chromosomal Variation in Lymphoblastoid Cell Lines. Hum. Mutat. 33 (7), 1075-1086. doi:10.1002/humu.22062

Sullivan, K. M., Mannucci, A., Kimpton, C. P., and Gill, P. (1993). A Rapid and Quantitative DNA Sex Test: Fluorescence-Based PCR Analysis of X-Y Homologous Gene Amelogenin. Biotechniques 15 (4), 636-638.

Tarasov, A., Vilella, A. J., Cuppen, E., Nijman, I. J., and Prins, P. (2015). Sambamba: Fast Processing of NGS Alignment Formats. Bioinformatics 31 (12), 2032-2034. doi:10.1093/bioinformatics/btv098

Taylor, J. C., Martin, H. C., Lise, S., Broxholme, J., Cazier, J. B., Rimmer, A., et al. (2015). Factors Influencing success of Clinical Genome Sequencing across a Broad Spectrum of Disorders. Nat. Genet. 47 (7), 717-726. doi:10.1038/ng. 3304

Thangaraj, K., Reddy, A. G., and Singh, L. (2002). Is the Amelogenin Gene Reliable for Gender Identification in Forensic Casework and Prenatal Diagnosis? Int. J. Leg. Med 116 (2), 121-123. doi:10.1007/s00414-0010262-y

Turro, E., Astle, W. J., Megy, K., Gräf, S., Greene, D., Shamardina, O., et al. (2020). Whole-genome Sequencing of Patients with Rare Diseases in a National Health System. Nature 583 (7814), 96-102. doi:10.1038/s41586020-2434-2

Webster, T. H., Couse, M., Grande, B. M., Karlins, E., Phung, T. N., Richmond, P. A., et al. (2019). Identifying, Understanding, and Correcting Technical Artifacts on the Sex Chromosomes in Next-Generation Sequencing Data. Gigascience 8 (7). doi:10.1093/gigascience/giz074

Ye, J., Coulouris, G., Zaretskaya, I., Cutcutache, I., Rozen, S., and Madden, T. L. (2012). Primer-BLAST: a Tool to Design Target-specific Primers for Polymerase Chain Reaction. BMC Bioinformatics 13, 134. doi:10.1186/14712105-13-134

Conflict of Interest: The authors declare that the research was conducted in the absence of any commercial or financial relationships that could be construed as a potential conflict of interest.

Publisher's Note: All claims expressed in this article are solely those of the authors and do not necessarily represent those of their affiliated organizations or those of the publisher, the editors, and the reviewers. Any product that may be evaluated in this article, or claim that may be made by its manufacturer, is not guaranteed or endorsed by the publisher.

Copyright (c) 2022 Liu, Zeng, Wang, Zhang, Chen, Wang, Wang, Lu, Guo and Bu. This is an open-access article distributed under the terms of the Creative Commons Attribution License (CC BY). The use, distribution or reproduction in other forums is permitted, provided the original author(s) and the copyright owner(s) are credited and that the original publication in this journal is cited, in accordance with accepted academic practice. No use, distribution or reproduction is permitted which does not comply with these terms. 